PROCEEDINGS OF THE

AMERICAN MATHEMATICAL SOCIETY

Volume 135, Number 4, April 2007, Pages 929-938

S 0002-9939(06)08726-0

Article electronically published on September 18, 2006

\title{
HENSELIAN VALUATIONS AND ORDERINGS OF A COMMUTATIVE RING
}

\author{
GUANGXING ZENG
}

(Communicated by Bernd Ulrich)

\begin{abstract}
The purpose of this paper is to investigate the interplay between henselian valuations and orderings (or semiorderings) of a ring. As a main result, it is proved that for a henselian valuation $v$ on a ring $R$, the following statements are equivalent: (1) $v$ is compatible with every semiordering of $R$; (2) $v$ is compatible with every ordering of $R$; (3) Every real prime ideal of $R$ is contained in the core of $v$.
\end{abstract}

\section{Introduction}

Valuation theory is an important tool in the study of real fields and ordered fields. As a main topic in the study of real fields and ordered fields, the interplay between valuations and orderings is investigated; see [7, 11. The close interplay between valuations and orderings of a field is based on the following definition.

Definition 0.1. Let $F$ be a field, let $v$ be a valuation on $F$, and $\leq$ an ordering of $F$. $\leq$ is said to be compatible with $v$, if $0 \leq a \leq b$ implies $v(a) \geq v(b)$ for $a, b \in F$.

When the valuation under consideration is henselian, the following result is well known (see Theorem 8.3 in 11]).

Theorem 0.2. Let $v$ be a henselian valuation on a field $F$. Then every semiordering of $F$ is compatible with $v$.

A general notion of valuations on a (not necessarily commutative) ring is due to Bourbaki [2. In this paper, only the smaller class of so-called Manis valuations is involved. In 1969, M. Manis 9] introduced the notion of valuations in the category of commutative rings. In the literature, these valuations introduced by M. Manis are hence called Manis valuations. Let $\Gamma$ be an ordered abelian group, written additively, and let $\Gamma_{\infty}=\Gamma \cup\{\infty\}$, where $\gamma+\infty=\infty+\gamma=\infty$ and $\gamma<\infty$ for all $\gamma \in \Gamma$. A map $v$ of a ring $R$ onto $\Gamma_{\infty}$ is called a Manis valuation on $R$, if $v(a b)=v(a)+v(b)$ and $v(a+b) \geq \min \{v(a), v(b)\}$ for all $a, b \in R$. In this case, $\Gamma$ is called the value group of $v$. For a valuation $v$ on $R$, we have the three subsets of $R$ as follows: $A_{v}:=\{a \in R \mid v(a) \geq 0\}, M_{v}:=\{a \in R \mid v(a)>0\}$, and $v^{-1}(\infty):=\{a \in R \mid v(a)=\infty\}$. It is easy to check that $A_{v}$ is a subring

Received by the editors October 23, 2005.

2000 Mathematics Subject Classification. Primary 13J30; Secondary 13J25, 12J15, 12D15.

Key words and phrases. Commutative ring, henselian valuation, ordering, real prime ideal.

This work was partially supported by a National Key Basic Research Project of China (Grant No. 2004CB318003).

(C)2006 American Mathematical Society Reverts to public domain 28 years from publication 
of $R, M_{v}$ is a prime ideal of $A_{v}, v^{-1}(\infty)$ is a prime ideal of both $R$ and $A_{v}$, and $v^{-1}(\infty) \subseteq M_{v} \subset A_{v}$. According to [9], such a pair $\left(A_{v}, M_{v}\right)$ is called a valuation pair of $R$ (precisely, the valuation pair of $v$ ), and $v^{-1}(\infty)$ is called the core of $v$. Let $v$ be a valuation on $R . v$ is called real if $A_{v} / M_{v}$ is a real domain, where $\left(A_{v}, M_{v}\right)$ is the valuation pair of $v$. It is clear that a valuation $v$ on a ring $R$ is real if and only if $v\left(a_{1}^{2}+\cdots+a_{n}^{2}\right)=2 \min \left\{v\left(a_{i}\right) \mid 1 \leq i \leq n\right\}$ for any $a_{1}, \ldots, a_{n} \in R$.

Many important facts about valuations on fields may be generalized to Manis valuations on commutative rings. Since the notion of orderings has been generalized to the category of commutative rings, it is a natural trend in real algebra to investigate the interplay between Manis valuations and orderings of commutative rings. In reference [10], M. Marshall discussed the compatibility of orderings with Manis valuations for commutative rings. As an important sort of valuations, henselian valuations were used to characterize semireal closed rings and real closed rings of higher level in references [13, 14. Naturally, such a question arises: Can Theorem 0.2 and other results on henselian valuations be generalized from the category of fields to that of commutative rings?

The main purpose of this paper is to establish several results on henselian valuations on a commutative ring so that Theorem 0.2 and other facts about henselian valuations on fields become their consequences.

Throughout this paper, "ring" means "commutative ring with identity element 1 ", "valuation on a ring" means "Manis valuation on a ring". Moreover, for two sets $A, B$, write $A \backslash B$ for the set $\{x \mid x \in A$ but $x \notin B\}$.

\section{Preliminaries}

In this section, we will give some definitions, and establish several basic results. First, let us recall the notion of positive cones of a ring. Let $R$ be a ring. A positive cone of $R$ is a subset $P$ of $R$ satisfying the following conditions: (1) $P+P \subseteq P$ and $P \cdot P \subseteq P,(2) R=P \cup-P$, and (3) $P \cap-P$ is a prime ideal of $R$. For generality of discussion, we further give the definition of semicones of a ring as follows.

Definition 1.1. Let $R$ be a ring. A semicone of $R$ is a subset $P$ of $R$ satisfying the following conditions:

(1) $1 \in P$.

(2) $P+P \subseteq P$, and $a^{2} P \subseteq P$ for all $a \in R$.

(3) $R=P \cup-P$.

(4) $P \cap-P$ is a prime ideal of $R$.

In the sequel, $P \cap-P$ is called the support of $P$ for a semicone $P$ of $R$. It is easy to see that $P \cap-P$ is a real prime ideal of $R$ if $P$ is a semicone of $R$.

Definition 1.2. Let $R$ be a ring. A binary relation $\leq$ on $R$ is called a semiordering (or ordering) of $R$, if the following subset $P_{\leq}$of $R$ is a semicone (or positive cone) of $R$ :

$$
P_{\leq}:=\{a \mid 0 \leq a\} .
$$

In what follows, the semicone (or positive cone) $P_{\leq}$of $R$ is called the semicone (or positive cone) of $\leq$.

Obviously, a positive cone (or ordering) is always a semicone (or semiordering) for a ring. However, by Theorem 7.17 in [11, a semiordering need not be an ordering even if the ring considered is a field. For a semiordering $\leq$ of $R$, it is easy to verify 
that $\leq$ satisfies reflexivity and transitivity, and either $a \leq b$ or $b \leq a$ for all $a$, $b \in R$. However, $\leq$ need not satisfy antisymmetry, even if $\leq$ is an ordering.

Let $P$ be a positive cone of $R$. Then, for $a \in R$, we may define the absolute value $|a|_{P}$ of $a$ with respect to $P$ such that $|a|_{P}=a$ if $a \in P$; otherwise $|a|_{P}=-a$. The absolute value $|\cdot|_{P}$ has properties similar to the usual absolute value of real numbers.

Based on the above definitions, the compatibility between semiorderings and valuations may be generalized to the category of commutative rings as follows.

Definition 1.3. Let $R$ be a ring, let $v$ be a valuation on $R$, and $\leq$ a semiordering of $R$. $\leq$ is said to be compatible with $v$, if $0 \leq a \leq b$ implies $v(a) \geq v(b)$ for $a$, $b \in R$. In this case, $P$ is also said to be compatible with $v$, if $P$ is the semicone of $\leq$.

For a valuation $v$ and a semicone $P$ of $R$, it is easy to see that $P$ is compatible with $v$ if and only if $v\left(p_{1}+p_{2}\right)=\min \left\{v\left(p_{1}\right), v\left(p_{2}\right)\right\}$ for all $p_{1}, p_{2} \in P$. Therefore, $P \cap-P \subseteq v^{-1}(\infty)$ whenever $P$ is compatible with $v$.

According to Definition 3 in [13], we give the following.

Definition 1.4. Let $R$ be a ring, let $v$ be a valuation on $R$ with valuation pair $(A, M)$, and denote by $\pi$ the canonical homomorphism of $A[x]$ onto $A / M[x] . v$ is called henselian if the following conditions are satisfied:

For any monic polynomial $f(x) \in A[x]$, if $\pi(f(x))=\phi(x) \psi(x)$, where $\phi(x)$ and $\psi(x)$ are two coprime monic polynomials in $A / M[x]$, then there exist monic polynomials $g(x), h(x)$ in $A[x]$ such that $f(x)=g(x) h(x), \pi(g(x))=\phi(x)$, and $\pi(h(x))=\psi(x)$.

Lemma 1.5. Let $R$ be a ring, and let $\leq$ be a semiordering of $R$. If $0 \leq a \leq b$, where $a, b \in R$, then $0 \leq a^{2} b \leq a b^{2}$.

Proof. From condition (1) in Definition 1.1, it follows that $0 \leq a^{2} b$. Moreover, we have $0 \leq a(a-b)^{2}+(b-a) a^{2}$ and $0 \leq a b^{2}-a^{2} b$. Hence $a^{2} b \leq a b^{2}$. This completes the proof.

Proposition 1.6. Let $R$ be a ring, let $v$ be a real valuation on $R$, and let $S$ be an integral extension of $R$. Then there is a real valuation on $S$ prolonging $v$, if and only if $S$ possesses a positive cone $Q$ such that $Q \cap R$ is compatible with $v$.

Proof. Assume that there is a real valuation $u$ on $S$ prolonging $v$. By a simple proof (see the proof of Theorem 2 in [3] ), $S$ possesses a positive cone $Q$ compatible with $u$. By Definition 1.3, it is clear that $Q \cap R$ is compatible with $v$.

Conversely, assume that $S$ possesses a positive cone $Q$ such that $Q \cap R$ is compatible with $v$. Denote by $\left(A_{v}, M_{v}\right)$ the valuation pair of $v$, put $P:=Q \cap R$, and put $\Delta:=\left(A_{v} \backslash M_{v}\right) \cap Q$. Obviously, $\Delta$ is closed under multiplication, i.e. $\delta_{1} \delta_{2} \in \Delta$ for all $\delta_{1}, \delta_{2} \in \Delta$. Since $Q \cap R$ is compatible with $v$, it is easy to prove that $\Delta$ is also closed under addition. Construct the two subsets of $S$ as follows:

$$
\begin{gathered}
A:=\left\{\left.x \in S\left|\delta_{1}\right| x\right|_{Q} \leq_{Q} \delta_{2} \text { for some } \delta_{1}, \delta_{2} \in \Delta\right\}, \\
M:=\left\{\left.x \in S\left|\delta_{1}\right| x\right|_{Q}<_{Q} \delta_{2} \text { for all } \delta_{1}, \delta_{2} \in \Delta\right\} .
\end{gathered}
$$

Obviously, $M \subset A$. It is routine to prove that $A$ is a subring of $S$ and $M$ is closed under addition. Let $a \in A$ and $y \in M$. Then $\delta_{1}|a|_{Q} \leq_{Q} \delta_{2}$ for some $\delta_{1}, \delta_{2} \in \Delta$. 
By the definition of $M, \delta_{2} \delta|y|_{Q}<_{Q} \delta_{1} \delta^{\prime}$ for all $\delta, \delta^{\prime} \in \Delta$. So we have the following inequality:

$$
\delta_{1} \delta|a y|_{Q} \leq_{Q} \delta_{2} \delta|y|_{Q}<_{Q} \delta_{1} \delta^{\prime} .
$$

Suppose $\delta_{1} \in Q \cap-Q$. Since $P$ is compatible with $v$, we get $\delta_{1} \in(Q \cap-Q) \cap R=$ $P \cap-P \subseteq v^{-1}(\infty) \subseteq M$, a contradiction. Hence $\delta_{1} \in Q \backslash(Q \cap-Q)$. By the previous equality, we have

$$
\delta|a y|_{Q}<_{Q} \delta^{\prime} \text { for all } \delta, \delta^{\prime} \in \Delta .
$$

By the definition of $M$, ay $\in M$. This implies that $M$ is an ideal of $A$. Let $a$, $b \in A \backslash M$. Then, there exist $\delta_{1}, \delta_{2}, \delta_{3}$ and $\delta_{4} \in \Delta$ such that $\delta_{1}|a|_{Q} \geq_{Q} \delta_{2}$ and $\delta_{3}|b|_{Q} \geq_{Q} \delta_{4}$. It follows that $\delta_{1} \delta_{3}|a b|_{Q} \geq_{Q} \delta_{2} \delta_{4}$. By the definition of $M, a b \notin M$. Therefore, $M$ is a prime ideal of $A$.

Now let $\alpha \in S \backslash A$. Since $S$ is integral over $R$, we have $\alpha^{n}+r_{1} \alpha^{n-1}+\cdots+r_{n}=0$, where $r_{i} \in R, i=1, \ldots, n$. Hence, the following statement is true:

There exists a natural number $m$ such that $\mid \alpha^{m}+r_{1} \alpha^{m-1}+\cdots+$ $\left.r_{m}\right|_{Q} \leq_{Q} \delta$ for some $\delta \in \Delta$ and $r_{i} \in R, i=1, \ldots, m$.

By the well-ordering property of natural numbers, the number $m$ may be assumed to be minimal. By the inequality in the above statement, we have

$$
\left|\alpha^{m}+r_{1} \alpha^{m-1}+\cdots+r_{m-1} \alpha\right|_{Q} \leq_{Q} \delta+\left|r_{m}\right|_{Q} .
$$

Suppose $r_{m} \in A_{v}$. Then $v\left(\left|r_{m}\right|_{Q}\right) \geq 0=v(\delta)$ and $v\left(\delta+\left|r_{m}\right|_{Q}\right)=\min \left\{v(\delta), v\left(\left|r_{m}\right|_{Q}\right)\right\}$ $=0$, since $P$ is compatible with $v$. Hence $\delta+\left|r_{m}\right|_{Q} \in \Delta$. Necessarily, $m>1$; otherwise $|\alpha|_{Q} \leq \delta+\left|r_{m}\right|_{Q}$ and $\alpha \in A$. Observe that $|\alpha|>_{Q} 1$ since $\alpha \notin A$. So we have $\left|\alpha^{m-1}+r_{1} \alpha^{m-2}+\cdots+r_{m-1}\right|_{Q} \leq_{Q}\left|\alpha^{m}+r_{1} \alpha^{m-1}+\cdots+r_{m-1} \alpha\right|_{Q} \leq_{Q}$ $\delta+\left|r_{m}\right|_{Q}$; this contradicts the minimality of $m$. Hence $r_{m} \notin A_{v}$. It follows that $v\left(\delta+\left|r_{m}\right|_{Q}\right)=\min \left\{v(\delta), v\left(\left|r_{m}\right|_{Q}\right)\right\}=v\left(r_{m}\right)<0$, i.e. $\delta+\left|r_{m}\right|_{Q} \notin A_{v}$. Therefore, there is an $a \in M_{v}$ such that $a\left(\delta+\left|r_{m}\right|_{Q}\right) \in A_{v} \backslash M_{v}$. Put $\delta_{1}:=|a|_{Q}\left(\delta+\left|r_{m}\right|_{Q}\right)$. Then $\delta_{1} \in \Delta$ and $|\beta \alpha|_{Q} \leq_{Q} \delta_{1}$, where $\beta:=a\left(\alpha^{m-1}+r_{1} \alpha^{m-2}+\cdots+r_{m-1}\right)$. By the definition of $A$, we have $\beta \alpha \in A$. On the other hand, we have

$$
\begin{aligned}
|\beta \alpha|_{Q} & =\left|a\left(\alpha^{m}+r_{1} \alpha^{m-1}+\cdots+r_{m-1} \alpha+r_{m}\right)-a r_{m}\right|_{Q} \\
& \geq_{Q}\left|a r_{m}\right|_{Q}-\left|a\left(\alpha^{m}+r_{1} \alpha^{m-1}+\cdots+r_{m-1} \alpha+r_{m}\right)\right|_{Q} \geq\left|a r_{m}\right|_{Q}-|a|_{Q} \delta .
\end{aligned}
$$

Observing that $v\left(\left|a r_{m}\right|_{Q}\right)=0<v\left(|a|_{Q} \delta\right)$, we have $\left|a r_{m}\right|_{Q}-|a|_{Q} \delta \in A_{v} \backslash M_{v}$. Moreover, by the compatibility of $P$ with $v$, it is easy to check that $\left|a r_{m}\right|_{Q}-|a|_{Q} \delta \in$ $P$. Hence $\left|a r_{m}\right|_{Q}-|a|_{Q} \delta \in \Delta$. By the definition of $M$, we have $\beta \alpha \notin M$. Suppose $\beta \notin M$. Then there exist $\delta_{2}, \delta_{3} \in \Delta$ such that $\delta_{2}|\beta|_{Q} \geq_{Q} \delta_{3}$. It follows that $\delta_{3}|\alpha|_{Q} \leq_{Q} \delta_{2}|\beta \alpha|_{Q} \leq_{Q} \delta_{2} \delta_{1}$. This yields $\alpha \in A$, a contradiction. Hence $\beta \in M$. This implies that $(A, M)$ is a valuation pair of $S$. Denote by $u$ the associated valuation of $(A, M)$. By the definition of $(A, M)$, it is clear that $Q$ is compatible with $u$. Thus $u$ is a real valuation on $S$. It is a routine to check $A \cap R=A_{v}$ and $M \cap R=M_{v}$. Therefore, $u$ is a prolongation of $v$. This completes the proof.

Proposition 1.7. Let $R$ be a ring, let $v$ be a real valuation on $R$, and let $S$ be an integral extension of $R$. If $S$ possesses a positive cone $Q$ such that $Q \cap R$ is compatible with $v$, then there is a unique real prolongation $u$ of $v$ on $S$ such that $Q$ is compatible with $u$. 
Proof. According to the proof of Proposition 1.6, there is a real prolongation $u$ of $v$ on $S$ such that $Q$ is compatible with $u$, and the valuation pair $(A, M)$ of $u$ is as in the proof of Proposition 1.6. It remains to prove the uniqueness of $u$.

Let $w$ be any real prolongation of $v$ on $S$ such that $Q$ is compatible with $w$. Denote by $\left(A_{w}, M_{w}\right)$ the valuation pair of $w$. Then we have the following.

Claim. $A \subseteq A_{w}, M_{w} \subseteq M$, and $A \backslash M \subseteq A_{w} \backslash M_{w}$.

Indeed, if $a \in A$, then $\delta_{1}|a|_{Q} \leq_{Q} \delta_{2}$ for some $\delta_{1}, \delta_{2} \in \Delta$, where $\Delta$ is as in the proof of Proposition 1.6. By the compatibility of $Q$ with $w$, we have $w\left(\delta_{1}|a|_{Q}\right) \geq w\left(\delta_{2}\right)$ and $w(a) \geq 0$. It follows that $a \in A_{w}$. Hence $A \subseteq A_{w}$. Suppose $M_{w} \not \subset M$. Then there is an $a \in M_{w}$ such that $a \notin M$. Then there exist $\delta_{1}, \delta_{2} \in \Delta$ such that $\delta_{1}|a|_{Q} \geq_{Q} \delta_{2}$. By the compatibility of $Q$ with $w$, we have $w\left(\delta_{1}|a|_{Q}\right) \leq w\left(\delta_{2}\right)$, and $w(a) \leq 0$, a contradiction. Hence $M_{w} \subseteq M$. From $A \subseteq A_{w}$ and $M_{w} \subseteq M$, it follows that $A \backslash M \subseteq A_{w} \backslash M_{w}$. The claim is verified.

By the above claim, we have $w^{-1}(\infty)=\left(M_{w}: S\right) \subseteq(M: S)=u^{-1}(\infty)$. Since both $u$ and $w$ are prolongations of $v, w^{-1}(\infty) \cap R=v^{-1}(\infty)=u^{-1}(\infty) \cap R$. By Corollary 5.9 in [1], $w^{-1}(\infty)=u^{-1}(\infty)$.

Let $\alpha \in A_{w}$. If $\alpha \in w^{-1}(\infty)$, then $\alpha \in u^{-1}(\infty) \subseteq A$. Now assume $\alpha \notin w^{-1}(\infty)$. Then $\alpha \notin u^{-1}(\infty)$. Since $\alpha$ is integral over $R$, we have

$$
a_{0} \alpha^{n}+a_{1} \alpha^{n-1}+\cdots+a_{n}=0,
$$

where $n \in \mathbb{N}, a_{0}=1$, and $a_{i} \in R, i=1, \ldots, n$.

Observe that

$$
\min \left\{u\left(a_{i} \alpha^{n-i}\right) \mid 0 \leq i \leq n\right\} \leq u\left(a_{0} \alpha^{n}\right)<\infty=u\left(a_{0} \alpha^{n}+a_{1} \alpha^{n-1}+\cdots+a_{n}\right) .
$$

Therefore, there are at least two $j, k$ in $\{0,1, \ldots, n\}$ with $j<k$ such that $u\left(a_{j} \alpha^{n-j}\right)$ $=u\left(a_{k} \alpha^{n-k}\right)=\min \left\{u\left(a_{i} \alpha^{n-i}\right) \mid 0 \leq i \leq n\right\}$. Obviously, $a_{j} \notin v^{-1}(\infty)$ and $a_{k} \notin$ $v^{-1}(\infty)$. Pick out one $b \in R$ such that $v(b)=v\left(a_{j}\right)-v\left(a_{k}\right)$. From $u\left(a_{j} \alpha^{n-j}\right)=$ $u\left(a_{k} \alpha^{n-k}\right)$, it follows that $u\left(b \alpha^{k-j}\right)=0$, so we have $b \alpha^{k-j} \in A \backslash M \subseteq A_{w} \backslash M_{w}$, and $w\left(b \alpha^{k-j}\right)=0$. This yields $-v(b)=w\left(\alpha^{k-j}\right) \geq 0$. On other hand, from $u\left(b \alpha^{k-j}\right)=0$ it follows that $u\left(\alpha^{k-j}\right)=-v(b) \geq 0$, and $u(\alpha) \geq 0$, i.e. $\alpha \in A$. Thus $A_{w} \subseteq A$. By the above claim, $A_{w}=A$.

It is similar to prove $M_{w}=M$. The proof is completed.

Lemma 1.8. Let $F$ be a field, let $K$ be an algebraic extension of $F$, and let $v$ be a valuation on $F$ such that $v$ possesses a unique prolongation $u$ on $K$. Then $v$ is henselian if and only if $u$ is henselian.

Proof. Obvious.

The following example shows that $\leq$ need not be compatible with $v$ for a henselian valuation $v$ and an ordering $\leq$ of a ring $R$. This is a difference between fields and rings.

Example 1.9. Let $x$ be an indeterminate over the field $\mathbb{Q}$ of rational numbers, and $R=\mathbb{Q}[x]$ the ring of polynomials in $x$ over $\mathbb{Q}$. Put $P:=\{f \in R \mid f(0) \geq 0\}$. Then $P$ is obviously a positive cone of $R$ such that $P \cap-P=x R$. Let $v$ be the trivial valuation on $R$ such that $v(0)=\infty$ and $v(f)=0$ for all non-zero $f \in R$. Then $(R,\{0\})$ is the valuation pair of $v$. Clearly, $v$ is a henselian valuation. Observe that $0 \leq_{P} x \leq_{P} 0$ but $v(x)<v(0)$. Hence $P$ is not compatible with $v$. 


\section{MAin RESUlts}

In this section, we shall establish several results on the close interplay between henselian valuations and semiorderings (or orderings) of a commutative ring, which may be regarded as certain generalizations of the corresponding facts about henselian valuations on a field.

Theorem 2.1. Let $R$ be a ring, let $v$ be a henselian valuation on $R$ with valuation pair $(A, M)$, and $I$ a real ideal of $R$. Then either $I \subseteq v^{-1}(\infty)$ or $M \subseteq I$.

Proof. Assume $I \nsubseteq v^{-1}(\infty)$. Then there exists an $a \in I$ such that $a \notin v^{-1}(\infty)$. In this case, there exists an $a^{\prime} \in R$ such that $a^{\prime} a \in A \backslash M$. For any $\xi \in M$, consider the polynomial $f_{\xi}(x):=x^{2}-\left(a^{\prime} a\right) x+\xi^{2}$. Denote by $\pi$ the canonical homomorphism of $A[x]$ onto $A / M[x]$. Obviously, $\pi\left(f_{\xi}(x)\right)=x^{2}-\overline{a^{\prime} a} x=x\left(x-\overline{a^{\prime} a}\right)$, where $\overline{a^{\prime} a}:=a^{\prime} a+M \in A / M$. Clearly, $x$ and $x-\overline{a^{\prime} a}$ are coprime. Since $v$ is a henselian valuation, $f_{\xi}(x)$ has a root $\alpha$ in $A$. It follows that $\alpha^{2}-a^{\prime} a \alpha+\xi^{2}=0$, so we have $\alpha^{2}+\xi^{2}=\left(a^{\prime} \alpha\right) a \in I$. Since $I$ is a real ideal of $R$, we have $\xi \in I$. Hence $M \subseteq I$. This completes the proof.

As a main result, we establish the theorem as follows.

Theorem 2.2. Let $R$ be a ring, and $v$ a henselian valuation on $R$. Then the following statements are equivalent:

(1) Every semiordering of $R$ is compatible with $v$.

(2) Every ordering of $R$ is compatible with $v$.

(3) Every real prime ideal of $R$ is contained in $v^{-1}(\infty)$.

Proof. (1) $\Longrightarrow(2)$ : Obvious.

$(2) \Longrightarrow(3)$ : Let $\wp$ be an arbitrary real prime ideal of $R$. It is well known that $R$ possesses a positive cone $P$ such that $P \cap-P=\wp$. For any $a \in \wp$, obviously $0 \leq_{P} a \leq_{P} 0$. By statement (2), $P$ is compatible with $v$. Hence $v(a) \geq v(0)=\infty$. Necessarily, $v(a)=\infty$, i.e. $a \in v^{-1}(\infty)$. By the arbitrariness of $a$, we have $\wp \subseteq v^{-1}(\infty)$.

$(3) \Longrightarrow(1)$ : Assume that every real prime ideal of $R$ is contained in $v^{-1}(\infty)$. Suppose that statement (1) is false. Then $R$ possesses at least one semiordering $\leq$ such that $\leq$ is not compatible with $v$. Therefore, there exist $a, b \in R$ such that $0 \leq a \leq b$ but $v(a)<v(b)$. Obviously, $v(a) \neq \infty$. Hence there is an $a^{\prime} \in R$ such that $v\left(a^{\prime}\right)=-v(a)$. It follows that $v\left(a^{\prime} a\right)=0$ but $v\left(a^{\prime} b\right)>0$. Write $(A, M)$ for the valuation pair of $v$. Then $a^{\prime} a \in A \backslash M$, but $a^{\prime} b \in M$. Consider the following polynomial in $A[x]$ :

$$
f(x)=x^{2}-\left(a^{\prime} a\right) x+a^{\prime 2} a b .
$$

Denote by $\pi$ the canonical homomorphism of $A[x]$ into $A / M[x]$. Observe that $a^{\prime 2} a b \in M$. Then $\pi(f(x))=x^{2}-\overline{a^{\prime} a} x=x\left(x-\overline{a^{\prime} a}\right)$, where $\overline{a^{\prime} a}:=a^{\prime} a+M \in A / M$. Clearly, $x$ and $x-\overline{a^{\prime} a}$ are coprime. Since $v$ is a henselian valuation, $f(x)$ has a root $\alpha$ in $A$. It follows that $\alpha^{2}-a^{\prime} a \alpha+a^{\prime 2} a b=0$ and $2 a^{\prime 2}\left(a^{2}-a b\right)=\left(\alpha-a^{\prime} a\right)^{2}+\alpha^{2}+\left(a^{\prime} a\right)^{2}$. Denote by $P$ the positive cone of $\leq$. Then

$$
2 a^{\prime 2}\left(a^{2}-a b\right) b=\left[\left(\alpha-a^{\prime} a\right)^{2}+\alpha^{2}+\left(a^{\prime} a\right)^{2}\right] b \in P .
$$

By Lemma $1.5, a^{2} b \leq a b^{2}$, i.e. $a^{2} b-a b^{2} \in-P$. This yields $2 a^{\prime 2}\left(a^{2}-a b\right) b=$ $2 a^{\prime 2}\left(a^{2} b-a b^{2}\right) \in-P$. So we have the following expression:

$$
2 a^{\prime 2}\left(a^{2}-a b\right) b=2 a^{\prime 2}\left(a^{2} b-a b^{2}\right) \in P \cap-P .
$$


Observe that $v\left(a^{\prime}\right)=-v(a) \neq \infty$, i.e. $a^{\prime} \notin v^{-1}(\infty)$. By statement (3), $P \cap-P \subseteq$ $v^{-1}(\infty)$, since $P \cap-P$ is a real prime ideal of $R$. This implies $a^{\prime} \notin P \cap-P$ and $2 a^{\prime 2} \notin P \cap-P$. Moreover, we may assert that $a^{2}-a b \notin P \cap-P$. Indeed, if not, then $\left(\alpha-a^{\prime} a\right)^{2}+\alpha^{2}+\left(a^{\prime} a\right)^{2}=2 a^{\prime 2}\left(a^{2}-a b\right) \in P \cap-P$. Since $P \cap-P$ is a real ideal, we get $a^{\prime} a \in P \cap-P \subseteq v^{-1}(\infty)$, a contradiction. By the above expression, we get $b \in P \cap-P$, since $P \cap-P$ is a prime ideal of $R$. Hence $a=(a-b)+b \in$ $(-P)+(-P) \subseteq-P$, and $a \in P \cap-P \subseteq v^{-1}(\infty)$, a contradiction. Therefore, statement (1) is true. The proof is completed.

By the proof of the above theorem, the following proposition can be obtained as an immediate consequence.

Proposition 2.3. Let $R$ be a ring, let $v$ be a henselian valuation on $R$, and $\leq a$ semiordering of $R$. Then $\leq$ is compatible with $v$ if and only if $P \cap-P \subseteq v^{-1}(\infty)$, where $P$ is the semicone of $\leq$.

For a ring $R$, a ring extension $S$ of $R$ is said to be a tight extension of $R$ if every non-zero ideal of $S$ contracts to a non-zero ideal of $R$. In reference [5], Enochs introduced the concept of a totally integrally closed ring to generalize the concept of an algebraically closed field. A ring $I$ is called a totally integrally closed ring if, for any ring homomorphism $\sigma: A \rightarrow I$ and any integral extension $B$ of $A$, there is a homomorphism of $B$ into $I$ extending $\sigma$. In order to apply the theory of totally integrally closed rings, the rings considered in the sequel are always assumed to be reduced rings, i.e. rings without non-zero nilpotent element. According to Theorem 2 in [5], every reduced ring $R$ possesses a tight integral extension, called the totally integral closure of $R$, which is totally integrally closed and is also unique up to $R$-isomorphism. Some concepts and results related to totally integrally closed rings can be found in [5] and [6].

As a generalization of real fields, we introduced the notion of real closed rings of higher level in the category of commutative rings; see Definition 2 in [14. It should be pointed out that the real closed rings of level 1 are different from those real closed rings, which were introduced by N. Sankaran and K. Varadarajan in 12. In what follows, we shall adopt the notion of real closed rings of higher level introduced in [14.

Let $v$ be a valuation on a ring $R$. Obviously, $v$ induces a valuation $\bar{v}$ on the domain $R / v^{-1}(\infty)$ such that $\bar{v}\left(a+v^{-1}(\infty)\right)=v(a)$ for all $a \in R$. In the sequel, $\bar{v}$ is called the valuation on $R / v^{-1}(\infty)$ induced by $v$. Obviously, the valuation pair of $\bar{v}$ is $\left(A / v^{-1}(\infty), M / v^{-1}(\infty)\right)$.

The following result is a generalization of Theorem 8.7 in [11] to the category of commutative rings.

Theorem 2.4. Let $R$ be a reduced ring, let $v$ be a real valuation on $R$ with valuation pair $(A, M)$, and $\bar{v}$ the valuation on $R / v^{-1}(\infty)$ induced by $v$ as above. If $A / v^{-1}(\infty)$ is integrally closed in its fraction field, then the following statements are equivalent:

(1) $\bar{v}$ is a henselian valuation.

(2) For every finite tight integral extension $S$ of $R$ and every prolongation $u$ of $v$ on $S$, every positive cone of $S$ with support $u^{-1}(\infty)$ is compatible with $u$.

Proof. Denote by $I(R)$ the totally integral closure of $R$, and write $F$ for the fraction field of $R / v^{-1}(\infty)$. Then, $F$ is also the fraction field of $A / v^{-1}(\infty)$, and a valuation 
$v^{*}$ on $F$ may be induced in the following way:

$$
\text { For } a \in R \text { and } b \in R \backslash v^{-1}(\infty), v^{*}\left(\frac{a+v^{-1}(\infty)}{b+v^{-1}(\infty)}\right)=v(a)-v(b) \text {. }
$$

Clearly, $v^{*}\left(\frac{a+v^{-1}(\infty)}{b+v^{-1}(\infty)}\right)=\bar{v}\left(a+v^{-1}(\infty)\right)-\bar{v}\left(b+v^{-1}(\infty)\right)$.

$(1) \Longrightarrow(2)$ : Assume that $\bar{v}$ is a henselian valuation on $R / v^{-1}(\infty)$. By the lemma on page 2179 in [13, $v^{*}$ is a henselian valuation.

Let $S$ be a finite tight integral extension of $R$, let $u$ be a prolongation of $v$ on $S$, and $P$ a positive cone of $S$ with support $u^{-1}(\infty)$. Since $u^{-1}(\infty) \cap R=v^{-1}(\infty)$, $R / v^{-1}(\infty)$ may be considered as a subring of $S / u^{-1}(\infty)$. Write $K$ for the fraction field of $S / u^{-1}(\infty)$, and denote by $u^{*}$ the valuation on $K$ induced by $u$ as $v^{*}$. Since $u^{-1}(\infty)$ is also the support of $P$, such a positive cone $P^{*}$ of $K$ may be obtained as follows:

For $a \in S$ and $b \in S \backslash u^{-1}(\infty), \frac{a+u^{-1}(\infty)}{b+u^{-1}(\infty)} \in P^{*}$, if and only if $a b \in P$.

Obviously, $K$ is a finite extension of $F$, and $u^{*}$ is a prolongation of $v^{*}$ on $K$. By Theorem 8.7 in [11, $P^{*}$ is compatible with $u^{*}$. Then, we may assert that $P$ is compatible with $u$. Indeed, if $0 \leq_{P} a \leq_{P} b$ for $a, b \in S$, then $0 \leq_{P^{*}} a+u^{-1}(\infty) \leq_{P^{*}}$ $b+u^{-1}(\infty)$, and $u(a)=u^{*}\left(a+u^{-1}(\infty)\right) \geq u^{*}\left(b+u^{-1}(\infty)\right)=u(b)$ since $P^{*}$ is compatible with $u^{*}$.

$(2) \Longrightarrow(1)$ : Since $v$ is a real valuation, $A / M$ is a real domain. Observe that the residue field of $v^{*}$ is the fraction field of $A / M$. Therefore, $v^{*}$ is a real valuation on F. By Corollary 7.10 in [11, there is at least one positive cone $P^{*}$ compatible with $v^{*}$. Construct the subset $P$ of $R$ as follows:

$$
P=\left\{a \in R \mid a+v^{-1}(\infty) \in P^{*}\right\} .
$$

It is easy to verify that $P$ is a positive cone of $R$ compatible with $v$ such that $P \cap-P=v^{-1}(\infty)$. By Proposition 1.1 in [14, the ordered ring $(R, P)$ admits a real closed ordered extension $(\widehat{R}, \widehat{P})$ (of level 1) such that $\widehat{R} \subseteq I(R)$. Put $\wp:=\widehat{P} \cap-\widehat{P}$. It follows that $\wp \cap R=P \cap-P=v^{-1}(\infty)$. Then $R / v^{-1}(\infty)$ may be viewed as a subring of $\widehat{R} / \wp$. Denote by $L$ the fraction field of $\widehat{R} / \wp$. Then $L$ is an algebraic extension of $F$. By a familiar fact about valuations on fields, $v^{*}$ may be prolonged to a valuation on $L$. Let $w^{*}$ be any prolongation of $v^{*}$ on $L$, and denote by $\Gamma$ the value group of $w^{*}$. Then, such a mapping $w$ of $\widehat{R}$ into $\Gamma \cup\{\infty\}$ may be obtained by defining $w(x)=w^{*}(x+\wp)$ for every $x \in \widehat{R}$. In order to verify that $w$ is a valuation on $\widehat{R}$, it is sufficient to prove that $w$ is surjective. Let $\gamma \in \Gamma$. Then $\gamma=w^{*}\left(\frac{\beta+\wp}{\alpha+\wp}\right)$, where $\alpha, \beta \in \widehat{R} \backslash \wp$. Since $\alpha$ is integral over $R$, we have

$$
a_{0} \alpha^{n}+a_{1} \alpha^{n-1}+\cdots+a_{n}=0,
$$

where $n \in \mathbb{N}, a_{0}=1$, and $a_{i} \in R, i=1, \ldots, n$.

According to the argument in the proof of Proposition 1.7, there are at least two $j, k$ in $\{0,1, \ldots, n\}$ with $j<k$ such that $w^{*}\left(a_{j} \alpha^{n-j}+\wp\right)=w^{*}\left(a_{k} \alpha^{n-k}+\wp\right)$, $w^{*}\left(a_{j}+\wp\right) \neq \infty$, and $w^{*}\left(a_{k}+\wp\right) \neq \infty$. Observe that $v\left(a_{j}\right)=v^{*}\left(a_{j}+v^{-1}(\infty)\right)=$ $w^{*}\left(a_{j}+\wp\right) \neq \infty$, and also $v\left(a_{k}\right) \neq \infty$. Pick out one $b \in R$ such that $v(b)=v\left(a_{j}\right)-$ $v\left(a_{k}\right)$. From $w^{*}\left(a_{j} \alpha^{n-j}+\wp\right)=w^{*}\left(a_{k} \alpha^{n-k}+\wp\right)$, it follows that $w^{*}\left(b \alpha^{k-j}+\wp\right)=0$. 
Hence

$$
\begin{aligned}
\gamma & =w^{*}(\beta+\wp)-w^{*}(\alpha+\wp)=w^{*}(\beta+\wp)+w^{*}\left(b \alpha^{k-j-1}+\wp\right) \\
& =w^{*}\left(b \alpha^{k-j-1} \beta+\wp\right)=w\left(b \alpha^{k-j-1} \beta\right),
\end{aligned}
$$

where $b \alpha^{k-j-1} \beta \in \widehat{R}$. Thus $w$ is surjective, and $w$ is a valuation on $\widehat{R}$. For every $a \in R, w(a)=w^{*}(a+\wp)=v^{*}\left(a+v^{-1}(\infty)\right)=v(a)$. Therefore, $w$ is a prolongation of $v$ on $\widehat{R}$. Clearly, $w^{-1}(\infty)=\wp$.

Now we proceed to prove that $\widehat{P}$ is compatible with $w$. Assume that $0 \leq_{\widehat{P}} \alpha \leq_{\widehat{P}}$ $\beta$, where $\alpha, \beta \in \widehat{R}$. Put $S:=R(\alpha, \beta), P_{S}:=\widehat{P} \cap S$, and $u:=\left.w\right|_{S}$ the restriction of $w$ to $S$. Then $S$ is a finite tight integral extension of $R$, and $P_{S}$ is a positive cone of $S$. Moreover, it is easy to see that $u$ is a prolongation of $v$ on $S$. Observe that $P_{S} \cap-P_{S}=\wp \cap S=w^{-1}(\infty) \cap S=u^{-1}(\infty)$. By statement (2), $P_{S}$ is compatible with $u$. So we have $u(\alpha) \geq u(\beta)$, i.e. $w(\alpha) \geq w(\beta)$. Hence $\widehat{P}$ is compatible with $w$. Naturally, $w$ is a real valuation on $\widehat{R}$, and $P(=\widehat{P} \cap R)$ is compatible with $v$.

According to Proposition 1.7, $w$ is uniquely determined by $v$ and $\widehat{P}$. Therefore, $w^{*}$ is also uniquely determined by $v$ and $\widehat{P}$, in other words, $w^{*}$ is the only prolongation of $v^{*}$ on $L$. By Theorem 1.1 and Proposition 1.2 in [14, $L$ is a real closed field. Since $w$ is a real valuation on $\widehat{R}, w^{*}$ is a real valuation on $L$. By Theorem 8.6 in 11, $w^{*}$ is henselian. By Lemma 1.8, $v^{*}$ is also a henselian valuation on $F$.

Now let $f(x)$ be a monic polynomial in $A / v^{-1}(\infty)[x]$, and assume that $\pi(f(x))=$ $\phi(x) \psi(x)$, where $\pi$ denotes the canonical homomorphism of $A / v^{-1}(\infty)[x]$ onto $\left(A / v^{-1}(\infty)\right) /\left(M / v^{-1}(\infty)\right)[x]$, and $\phi(x)$ and $\psi(x)$ are two coprime monic polynomials in $\left(A / v^{-1}(\infty)\right) /\left(M / v^{-1}(\infty)\right)[x]$. Denote by $A_{v^{*}}$ the valuation ring of $v^{*}$, and $M_{v^{*}}$ the maximal ideal of $A_{v^{*}}$. Then, $\left(A / v^{-1}(\infty)\right) /\left(M / v^{-1}(\infty)\right)$ may be viewed as a subring of $A_{v^{*}} / M_{v^{*}}$. Since $v^{*}$ is henselian, there exist monic polynomials $g(x)$, $h(x)$ in $A_{v^{*}}[x]$ such that $f(x)=g(x) h(x), \pi^{*}(g(x))=\phi(x)$, and $\pi^{*}(h(x))=\psi(x)$, where $\pi^{*}$ is the canonical homomorphism of $A_{v^{*}}[x]$ onto $A_{v^{*}} / M_{v^{*}}[x]$.

Denote by $\Omega$ the algebraic closure of $F$. Then $f(x)$ may be factored as follows: $f(x)=\left(x-\alpha_{1}\right) \cdots\left(x-\alpha_{n}\right)$, where $\alpha_{i} \in \Omega, i=1, \ldots, n$. So we may assume $g(x)=\left(x-\alpha_{1}\right) \cdots\left(x-\alpha_{s}\right)$ and $h(x)=\left(x-\alpha_{s+1}\right) \cdots\left(x-\alpha_{n}\right)$, where $0 \leq s \leq n$. Since $\alpha_{i}$ is integral over $A / v^{-1}(\infty)$ for $i \in\{1, \ldots, n\}$, all the coefficients of both $g(x)$ and $h(x)$ are integral over $A / v^{-1}(\infty)$. By the hypothesis that $A / v^{-1}(\infty)$ is integrally closed in $F$, we have $g(x), h(x) \in A / v^{-1}(\infty)[x]$. Since $\pi$ is the restriction of $\pi^{*}$ to $A / v^{-1}(\infty)[x]$, we have $\pi(g(x))=\pi^{*}(g(x))=\phi(x)$, and $\pi(h(x))=\pi^{*}(h(x))=\psi(x)$. Hence $\bar{v}$ is a henselian valuation on $R / v^{-1}(\infty)$. This completes the proof.

Corollary 2.5. Let $R$ be a reduced ring such that $1+a_{1}^{2}+\cdots+a_{n}^{2}$ is invertible for every natural number $n$ and all $a_{1}, \ldots, a_{n} \in R$, let $v$ be a real valuation on $R$, and $\bar{v}$ the valuation on $R / v^{-1}(\infty)$ induced by $v$ as above. If all positive cones of $S$ are compatible with u for every finite tight integral extension $S$ of $R$ and every prolongation $u$ of $v$ on $S$, then $\bar{v}$ is a henselian valuation.

Proof. Denote by $(A, M)$ the valuation pair of $v$. According to the proof of Theorem 2.4 , it is sufficient to prove that $A / v^{-1}(\infty)$ is integrally closed in its fraction field $F$. Suppose that $A / v^{-1}(\infty)$ is not integrally closed in $F$. Then there exists an $\alpha \in F$ such that $\alpha$ is integral over $A / v^{-1}(\infty)$ but $\alpha \notin A / v^{-1}(\infty)$. Since $A / v^{-1}(\infty)$ 
is integrally closed in $R / v^{-1}(\infty)$, it follows that $\alpha \notin R / v^{-1}(\infty)$. Now consider the following ideal $J$ of $R$ :

$$
J:=\left\{a \in R \mid\left(a+v^{-1}(\infty)\right) \alpha \in R / v^{-1}(\infty)\right\}
$$

Obviously, $v^{-1}(\infty) \subset J$. For any $a_{1}, \ldots, a_{n} \in R$, we have $1+a_{1}^{2}+\cdots+a_{n}^{2} \notin J$, since $\alpha \notin R / v^{-1}(\infty)$ and $1+a_{1}^{2}+\cdots+a_{n}^{2}$ is invertible. This implies that $J$ is a semireal ideal of $R$. By Theorem 2.3 in 8 and its proof, there is a real prime ideal $I$ of $R$ such that $J \subseteq I$. By the hypothesis, every positive cone of $R$ is compatible with

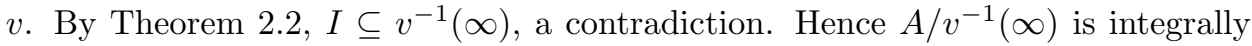
closed in its fraction field. The proof is completed.

\section{REFERENCES}

1. M. F. Atiyah and I. G. Macdonald, Introduction to Commutative Algebra, Addison-Wesley, Reading, MA, 1969. MR.0242802 (39:4129)

2. N. Bourbaki, Algébre commutative, Hermann, Paris, 1964. MR0194450 (33:2660)

3. Z. Dai, Real places and real valuations on a commutative ring, Acta Math. Sinica, Special Issue, 10(1994), 14-25. MR.1268256 (95b:13003)

4. O. Endler, Valuation Theory, Springer-Verlag, New York, 1972. MR0357379 (50:9847)

5. E. Enochs, Totally integrally closed rings, Proc. Amer. Math. Soc. 19(1968), 701-706. MR.0224600 (37:199)

6. M. Hochster, Totally integrally closed rings and extremal spaces, Pacific J. Math. 32(1970), 767-779. MR0257064 (41:1718)

7. T. Y. Lam, The Theory of Ordered Fields, Lecture Notes in Pure and Appl. Math. 55, M. Dekker, New York, 1980. MR0584611 (82e:12033)

8. T. Y. Lam, An introduction to real algebras, Rocky Mountain J. Math. 14(1984), 767-814. MR.0773114 (86g:12013)

9. M. Manis, Valuations on a commutative ring, Proc. Amer. Math. Soc. 20(1969), 193-198. MR0233813 (38:2134)

10. M. Marshall, Orderings and real places on a commutative ring, J. Algebra 140(1991), 484-501. MR:1120436 (92g:13030)

11. A. Prestel, Lecture on formally real fields, Lecture Notes in Math. 1093, Springer-Verlag, Berlin, New York, 1984. MR0769847(86h:12013)

12. N. Sankaran and K. Varadarajan, Formally real rings and their real closures, Acta Math. Hungarica 70(1996), 101-120. MR1361464 (96h:13022)

13. G. Zeng, On semireal closed rings, Comm. in Algebra 29(2001), 2171-2183. MR.1837969 (2002f:13050)

14. G. Zeng, On real closed rings of higher level, J. Algebra 290(2005), 250-265. MR2154992

Department of Mathematics, Nanchang University, Jiangxi Province, Nanchang 330047, People's Republic of China

E-mail address: zenggx@ncu.edu.cn 